\title{
Influence of stiffness constraints on optimal design of trusses using morphological indicators
}

\author{
T. Vandenbergh ${ }^{1}$, W. P. De Wilde ${ }^{1}$, P. Latteur ${ }^{2}$, B. Verbeeck ${ }^{1}$, \\ W. Ponsaert ${ }^{1} \&$ J. Van Steirteghem ${ }^{1}$ \\ ${ }^{I}$ Faculty of Engineering Sciences, \\ Department of Mechanics of Materials and Structures, \\ Vrije Universiteit Brussels, Brussels, Belgium \\ ${ }^{2}$ Agronomic Faculty of Gembloux and Setesco NV, Belgium
}

\begin{abstract}
Within the framework of sustainable development we strive for structures with a minimum volume of material. When we only consider criteria on resistance and buckling, Samyn and Latteur prove that even at the stage of conceptual design a clear hierarchy among the different truss typologies can be established. Up to now, stiffness constraints - such as the upper limit on static displacements - were not considered. However, an optimum obtained by minimising the volume, only considering the strength criterion, often results in solutions which violate the stiffness constraint(s). To avoid large displacements a stress level reduction can be imposed. However, this comes at the cost of a significant volume increase. With an optimisation process that involves the stiffness constraints at the stage of conceptual design, an optimum can be obtained without the necessity to alter the structure drastically afterwards, which partly annihilates the main objective of minimal use of material. This approach compares the different truss types on a new priority scale, generating new optima. This implicates a non-negligible change in the truss choice at conceptual design stage. The solutions are logically depended on the displacement criterions. This approach forms a first step to a new design philosophy that considers all the stiffness constraints (static displacements, resonance, local and global buckling) at conceptual design stage and is called design for stiffness.

Keywords: morphological indicators, stiffness, strength, truss, steel, static displacements, optimisation.
\end{abstract}




\section{Introduction}

\subsection{Morphological indicators}

Morphological Indicators (MI) are design tools allowing the optimisation of structures for a chosen criterion (volume, stiffness) at the stage of conceptual design using a limited number of parameters [1]. The indicator of volume $W=\sigma V / F L$ allows the comparison of the volume of material used of different structural systems. It is the volume of an isomorphic structure with unit span $L$, with at least one section dimensioned on its unit allowable stress $\sigma$, subjected to a system of loads with unit resultant $F$. The displacement indicator $\Delta=E \delta / \sigma L$ compares the displacement of different structural systems. It is the maximum displacement of an isomorphic structure with unit $\operatorname{span} L$ in a material with unit Young's modulus $E$, with at least one section dimensioned on its unit allowable stress $\sigma$, subjected to a system loads with unit resultant $F$.

The analytical expressions of both $W$ and $\Delta$ have been established by Samyn [1] and Latteur [2] for trusses, beams, arches, cables, cable stayed structures, masts and frames subjected to a limited number of (simple) load cases and supports.

For statically determined structures those MI are only function of the geometrical slenderness $L / H$ if instabilities, self weight and second order effects are neglected. Efficiency curves depicting the geometrical slenderness with respect to minimum volume material can be established (Figure 1).

The indicator of buckling $\Psi=\mu \sigma L / \sqrt{q E F}$ is developed by Latteur [2] to take into account buckling in compression elements. It is the image of the buckling tendency of the compression elements in a structure with span $L$, composed of bars with a form factor $q=I / \Omega^{2}$ (with $I$ the moment of inertia and $\Omega$ the section area) in a material with Young's modulus $E$, with at least one section dimensioned on its allowable stress $\sigma$ with a system of load with total resultant $F . \mu$ is the proportion of the buckling length of the compression bars over their geometrical length (which depends on the connection type).

\subsection{Stiffness constraints}

Up to now, stiffness constraints have never been considered in the use of MI. Only the resistance criterion was met with volume as the objective function. This strategy is called design for strength and often results in lightweight structures with a problematic lack of stiffness, which implicates a non-negligible volume increase to meet the (imposed) stiffness criteria. In this paper we only consider one stiffness constraint: the upper limit on the static displacement. We develop an optimisation process that involves this stiffness constraint at the stage of conceptual design and obtain an optimum without the necessity to change the structure drastically afterwards.

Taking stiffness into account compares the different truss types on a new priority scale, generating new optima. This implicates a non-negligible change in 
the truss choice at conceptual design stage. As expected, we show that the solutions are dependent on the displacement criteria.

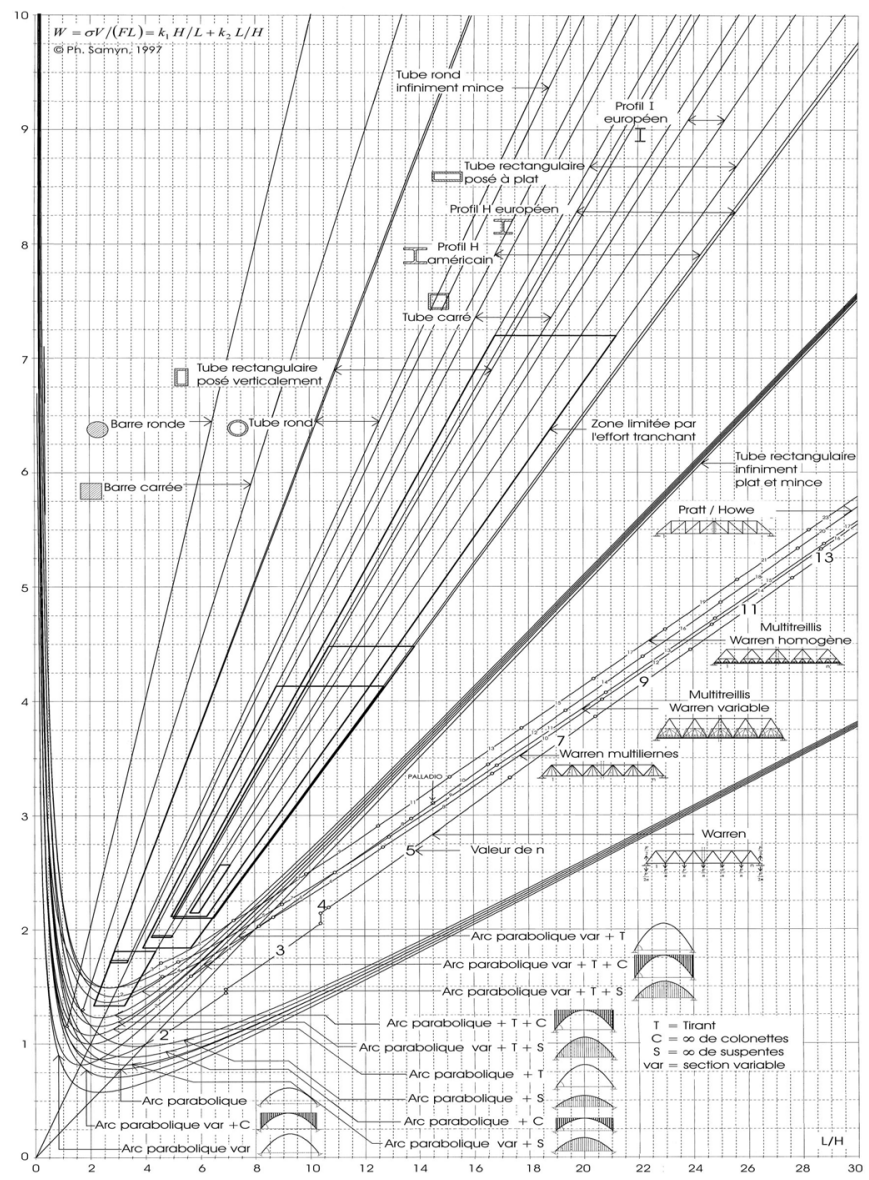

Figure 1: Indicator of volume as a function of the slenderness for some classical structure typologies, neglecting buckling, Samyn [1].

\section{Upper limit on static displacements}

In Eurocode 3 [4] we can find the normative constraint on static displacements. This is usually expressed as an upper limit on $\delta / L . \delta$ is the maximum total displacement of the structure and $L$ the span of the structure. Those are only indicative values; the commissioner of the project can impose sterner limits. 
There are 2 types of constraints: those on the displacements due to the total loads and those on the displacements due to the live loads only. The determinative criterion depends on the ratio of live loads to the total loads (self weight included). We can demonstrate that when this percentage is smaller than $74 \%$ we must consider the constraints on the static displacements due to the total loads. Since this is almost always the case, from now on we only consider the displacement constraints due to the total loads.

Considering the nature of the displacement constraints, those can very easily be translated to an upper limit on the displacement indicator, $\Delta$. Imposing an upper value on $\delta / L$ results in imposing an upper value on $\Delta$. However the constraint on $\Delta$ becomes material dependant. The larger the proportion of Young's modulus over the allowable stress, the larger the allowable value of $\Delta$ and the less severe the constraint. Table 1 shows the basic material properties of the classic structure steels.

Table 1: $\quad$ Material properties of (common types of) structure steel.

\begin{tabular}{|c|c|c|c|c|c|}
\hline Name & $\begin{array}{c}\text { Specific } \\
\text { weight } \rho \\
\left(\mathrm{N} / \mathrm{m}^{3}\right)\end{array}$ & $\begin{array}{c}\text { Young's } \\
\text { modulus } E \\
(\mathrm{GPa})\end{array}$ & $\begin{array}{c}\text { Allowable } \\
\text { strength } \sigma \\
(\mathrm{MPa})\end{array}$ & $E / \sigma$ & $\sigma / \rho(\mathrm{m})$ \\
\hline $\mathrm{S} 235$ & 78500 & 210 & 235 & 894.6 & 2993.6 \\
\hline $\mathrm{S} 275$ & 78500 & 210 & 275 & 763.6 & 3503.2 \\
\hline $\mathrm{S} 355$ & 78500 & 210 & 355 & 591.5 & 4522.3 \\
\hline
\end{tabular}

Finally it is important to mention that, according to the Eurocodes, a calculation of static displacements is a Serviceability Limit State (SLS) calculation as opposed to a strength calculation which is an Ultimate Limit State (ULS) one. The difference between, SLS and ULS resides in the partial safety coefficient imposed on the loads: in ULS we typically use 1.5 for live loads and 1.35 for permanent loads, in SLS, on the other hand 1.0 for both load types. At conceptual design stage, we can accurately transform loads in ULS to SLS by dividing the total loads in ULS by 1.4 to obtain the total loads in SLS. Since the static displacements are linearly proportional to the loads, we must divide the displacement indicator calculated in Samyn [1] and Latteur [2] by 1.4.

\section{Indicator of volume of structures subjected to stiffness constraint}

\subsection{Strategy}

We violate the displacement constraint for a large interval of the slenderness and for common typologies of trusses. Figure 2 depicts the indicator of displacement $\Delta$ for Warren trusses with minimal $W$. We observe that for common values of $\delta / L(1 / 200$ and 1/500) an upper value for $\Delta$ exists which we cannot exceed. This value of $\Delta$ determines a validity interval for the slenderness. 


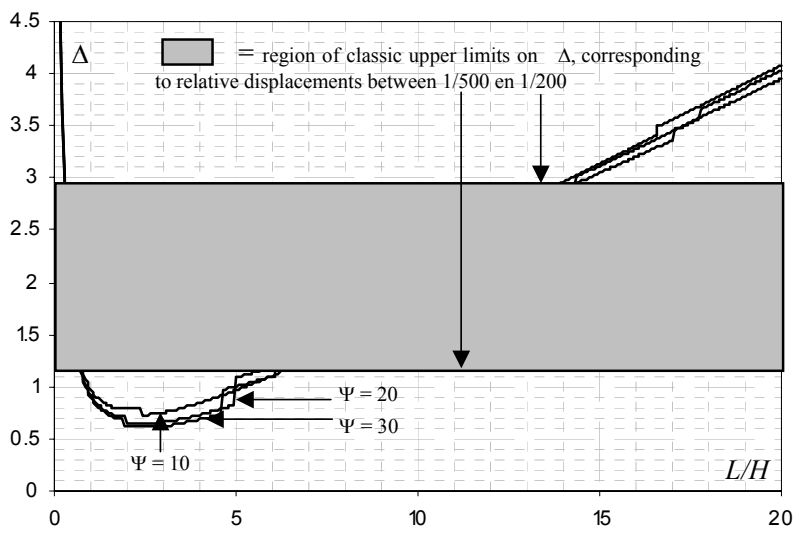

Figure 2: Displacement indicator of the lightest Warren trusses and common, displacement constraints for $S 355$ steel.

The designer is presented with different solutions:

- Change the slenderness. This often results in a smaller slenderness. However, in most cases a maximal height is imposed which results in a minimum slenderness

- Change the typology (number of meshes and/or truss type). This solution is also subjected to technological and architectural limitations.

- Introduce an initial camber: this can compensate the displacement caused by the permanent loads.

- Reduce the stress level: by doing so we increase the stiffness of the structure. However this comes at the cost of an increase in volume of material.

The scope of these solutions and their respective impact on the increase of volume of material must be considered at conceptual design stage. A possible approach is the calculation of $W$ and $\Delta$ for every structure. Then we must compare the calculated indicator of displacement to its imposed upper limit. When the calculated displacement is allowable we use this structure. If, on the other the hand we violate the constraint we impose a stress reduction to increase the stiffness and meet the criterion on static displacements. We use $\beta$ to denote the necessary stress reduction and define $\beta$ as the ratio of the applied stress level to the maximum allowable stress level.

Finally we determine the indicator of volume for this reduced stress level. This leads to a relatively accurate prediction of the volume of the lightest structure considering the strength requirement and the constraints on static displacements. The 'new' optimum curves depend on these stiffness constraints and therefore on $\delta / L$ and the material of the structure. The number of parameters increases from $2(\Psi$ and $L / H)$ to $4(\Psi, L / H, \delta / L$ and material). The main disadvantage is that we cannot draw general design curves valid for all structures 
as those set up in Samyn [1] and Latteur [2]. Nevertheless, the number of parameters remains small being the main advantage of the MI.

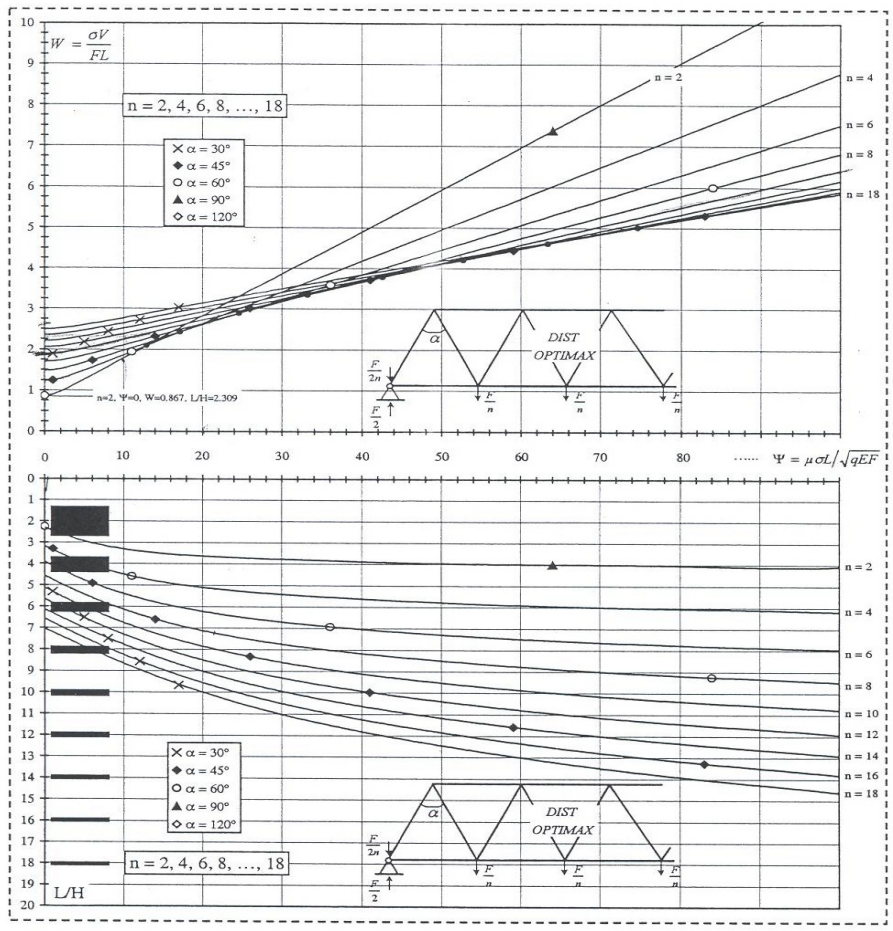

Figure 3: Optimum indicator of volume and corresponding slenderness as a function of the indicator of buckling for Warren truss loaded on their lower chord [2].

\subsection{Results}

In [2] Latteur selects the optimal (i.e. the lowest) indicator of volume $W$ and its corresponding slenderness $L / H_{\text {opt }}$ for every value of the indicator of buckling $\Psi$. We plot $W$ and $L / H$ as a function of $\Psi$ (Figure 3). This allows us to determine the optimal value of $W$ and $L / H$ if $\Psi$ is known. However, the main disadvantage is the absence of sensitivity! Those curves only show the optimal values. For another value of the slenderness, no information is available. Considering that most optima are found for slendernesses between 2 and 12 [2] and that usually trusses have values of the slenderness between 8 and 18 [3], working at the optimum values is not always possible. Therefore we clearly divide the input information in variables and parameters. The material parameters and the displacement constraint are considered as being input parameters, since their values are fixed. For this set of parameters, we plot 
efficiency curves for the indicator of volume as function of the slenderness for $\Psi=0,10,20,30 . \quad \Psi=0$ corresponds to the theoretical value of Samyn [1], in which buckling is neglected. Common values of $\Psi$ vary between 10 and 30 . Between the curves of $\Psi=10,20$ and 30 linear interpolation is allowed, certainly at conceptual design stage.
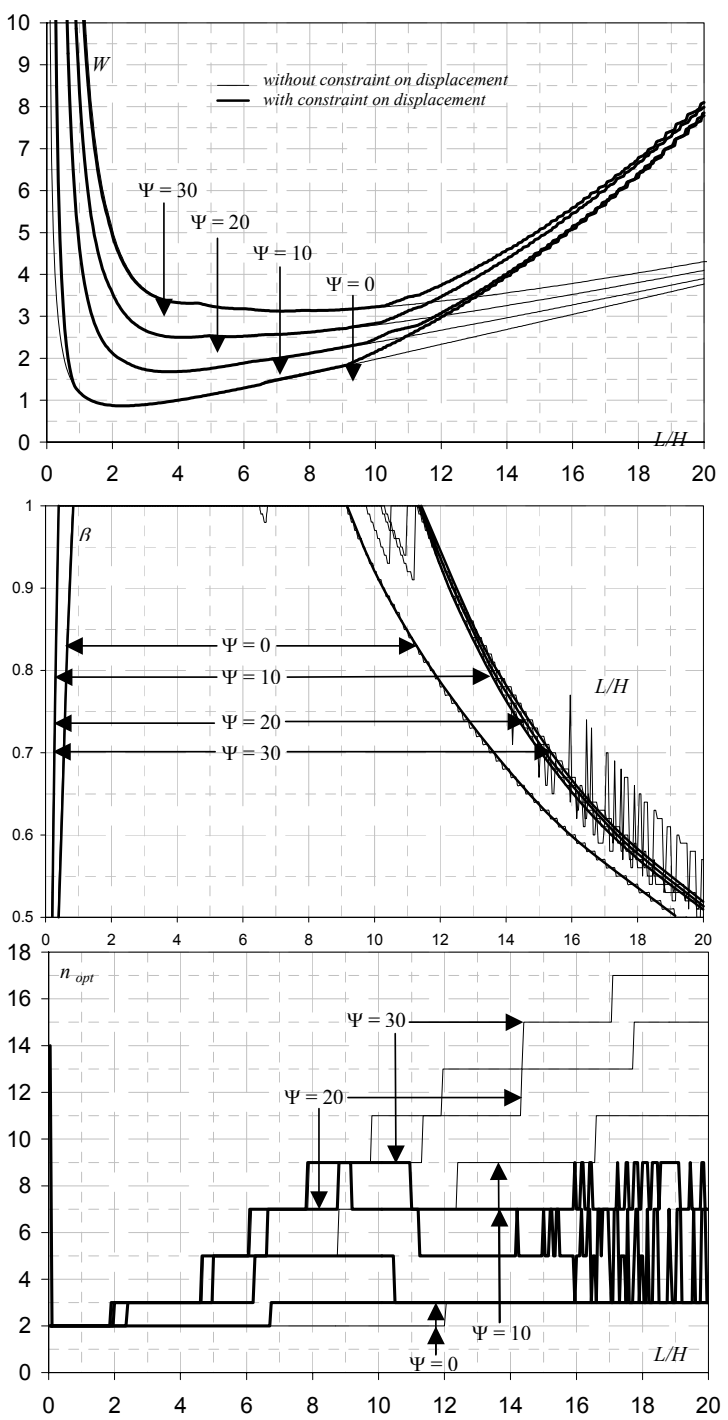

Figure 4: Design curves of Warren truss of $S 355$ steel loaded on their lower chord, with (bold) and without the upper limit on static displacement of $1 / 300$. 
We also provide the optimal stress level reduction as a function of the slenderness for the same values of $\Psi$, illustrating the impact of the stiffness constraint on the 'exhaustion level' of the elements.

To limit the amount of curves those calculations are repeated for values of the slenderness between 0 and 20 and $n$ (number of meshes) between 2 to 18 . Only the trusses with optimal (minimum $W$ ) number of meshes $n_{\text {opt }}$, are selected. Finally, for every slenderness, the corresponding $n_{\text {opt }}$ is plotted, representing the optimal truss.

This yields 3 design graphs for each set of input parameters (Figure 4, bold curves). The noise is the result of numerical calculations. A corresponding trend line indicates clearly the tendency.

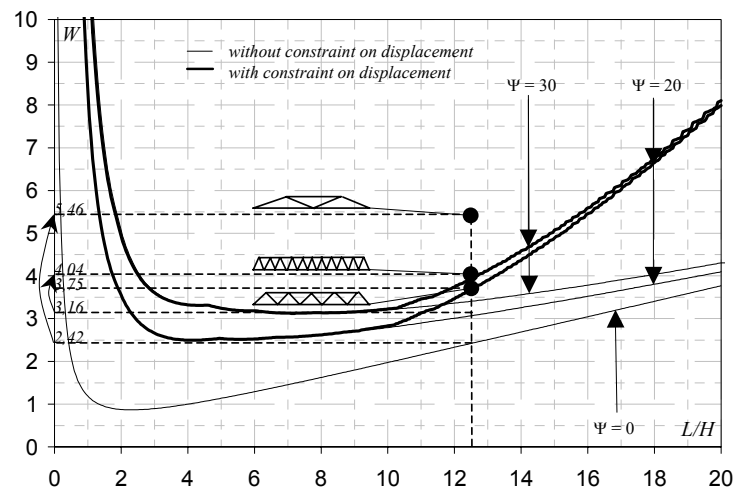

Figure 5: Impact of design for stiffness versus design for strength on optimisation of volume.

\section{Practical example}

We design a $S 355$ steel Warren truss with a span of $50 \mathrm{~m}$ and a maximal height of $4 \mathrm{~m}$. We must limit the maximal static displacement to $1 / 300$. If we only consider the resistance criterion [1] the slenderness and the mesh number are the only variables and we obtain a truss with a slenderness of 12,5 and 2 meshes when we limit the volume of material. In the strategy of Latteur [2], in which buckling is considered explicitly, more input information is needed to determine the value of $\Psi$ : the selected material, the section type of the bars and the total load. $S 355$ steel, tubes with thickness/diameter ratio of 0.04 and a total load of $2500 \mathrm{kN}$, result in an indicator of buckling of $\Psi=22.62$. We obtain an optimum truss with 11 panels and a slenderness of 12,5. Finally, the constraint on the static displacement does not influence the slenderness, on the other hand the number of panels decreases to 5 . For this truss we obtain $W=3.75$. Since we include more phenomena (buckling, static constraints), the indicator of volume obtained by Samyn [1] increases (from 2.42 to 5.46). The solution obtained by 
Latteur [2] also increases due to the constraint on the static displacement (from 3.16 to 4.04 ). Those results are shown in Figure 5 and clearly illustrate the importance of considering stiffness constraints at conceptual design stage. Here we obtain a gain in volume of $8 \%$ in comparison with Latteur [2] and up to $46 \%$ with the result of Samyn [1].

\section{Conclusions}

We found that trusses with a large stiffness are composed of a small number of panels. Even though a larger number of panels reduces the buckling length of compression members, we observe that when we consider the constraint on static displacements we obtain an optimal solution with a smaller number of panels [1].

Moreover, stiff trusses are usually stocky (typically $L / H$ between 1.5 and 5), which confirms the results obtained by Samyn [1], guiding us towards small slendernesses. We observe that these stiff trusses are often very light (especially when the truss is not sensitive to buckling).

The more buckling sensitive the truss, the smaller the necessary stress level reduction, since the material against buckling also provides stiffness against displacements. This can be noticed on the curves by the larger $\beta$ 's at larger $\Psi ' s$ for the same slenderness. On the other hand, a stress level reduction against displacements means a less buckling sensitive truss. This explains why at larger slendernesses (the most compliant geometry), the curves of the different $\Psi$ 's join. For very large $L / H$ 's and/or very strict displacements constraints, we can assume that the optimal structures become independent of $\Psi$.

The optimal stress level is not always $\beta=1$. This clearly shows that fully stressed design doesn't always give the lightest structure.

Finally, Howe and Pratt trusses do not present any advantage in comparison with Warren trusses, since they not only need more material but are less stiff.

\section{Further research}

The above mentioned strategy, which considers the constraints on static displacements, can be applied to other truss topologies (K, Long, Smith,...) and to different typologies (arches, beams...). Hence, we can consider a new design hierarchy with respect to the minimisation of volume

This approach constitutes a first step to a design philosophy that considers all stiffness constraints (static displacements, resonance, local and global buckling) at conceptual design stage, called design for stiffness.

Finally, we must consider the influence of the connections (fixed or pinned) on the stiffness and on the volume of material.

\section{Acknowledgement}

I would like to acknowledge the Institute for the Promotion of Innovation through Science and Technology in Flanders (IWT Vlaanderen), which helps this research financially. 
40 High Performance Structures and Materials III

\section{References}

[1] Samyn P., Etude Comparée du Volume et du déplacement de Structures Isostatiques Bidimensionnelles sous Charges Verticales entre Deux Appuis. Vers un outil d'évaluation de prédimensionnement des structures (Tome 1 à 4), $\mathrm{PhD}$ thesis, Université de Liège, Belgium, 1999.

[2] Latteur P., Optimisation et Prédimensionnement des Treillis, Arcs, Poutres et Câbles sur Base d'Indicateurs Morphologiques (Tome 1 à 4), $\mathrm{PhD}$ thesis, Vrije Universiteit Brussel, Belgium, 2000.

[3] Orton A., The way we build now (Chapter 2). Building Structures, E \& FN Spon , pp. 42-44, 1994.

[4] Eurocode Steel/3, NBN ENV 1993-1-1:1992 pp. 49-52, 1992. 